\title{
Mobilizing pre-heart-transplant patients with a percutaneously placed axillary-subclavian intraaortic balloon pump: A retrospective study
}

\author{
Frederick R. Macapagal, Lisa Green, Emma McClellan, Carrie Bridges \\ Houston Methodist Hospital, Houston, Texas, United States
}

Received: June 22, 2017

Accepted: September 28, 2017 Online Published: December 17, 2017

DOI: $10.5430 /$ jnep.v8n5p1

URL: https://doi.org/10.5430/jnep.v8n5p1

\begin{abstract}
The intraaortic balloon pump (IABP) is a treatment for end-stage heart failure patients not responsive to pharmacological therapy pre heart transplantation. An alternative approach-the percutaneously placed axillary-subclavian intraaortic balloon pump (PAxIABP) developed by cardiologists in our hospital enable patients to be mobilized while awaiting transplant versus the traditional bedrest in the femoral approach. Our objective is to determine if PAxIABP therapy enable pre-heart-transplant patients to safely mobilize. A retrospective study of pre-heart-transplant PAxIABP patients in the Coronary Intensive Care Unit (CICU) from 2007 to 2013 ( $n=45 ; 35$ men, 10 women) was conducted to determine mobility. Data are presented as mean (standard deviation) for continuous variables and number (percentage) for categorical variables. Patients were mobilized at $1.39( \pm 1.41)$ days after PAxIABP insertion. The number of times mobilized per day was $1.79( \pm 2)$. Transplant waiting time was $24.98( \pm$ $25.03)$ days. Thirty-seven patients $(82 \%)$ were transplanted. Two patients had a left ventricular assist device (LVAD) inserted and later transplanted. Six patients died before receiving a heart transplant, patients were 100\% mobile. Complication rates: $4 \%$ bleeding; 7\% ischemic complications; 2\% IABP-related infection. Pre-heart-transplant patients receiving PAxIABP therapy can be safely mobilized. Nursing care protocols were developed to safely take care of this patient population. PAxIABP can be utilized for other procedures requiring long IABP therapy.
\end{abstract}

Key Words: Functional independence measure, Intraaortic balloon pump, Heart failure, Heart transplant, Mobilization

\section{INTRODUCTION}

Heart failure is a clinical syndrome combination of shortness of breath and poor exercise tolerance. ${ }^{[1]}$ Heart failure is a chronic, debilitating disease that often progresses quickly to end-stage when severe left ventricular dysfunction leads to alterations in organ perfusion even at rest. Heart failure prevalence in Americans 20 years old and above had increased to 6.5 million (2011-2014), compared to 5.7 million from 2009-2012. ${ }^{[2]}$ Heart transplantation is the definitive treatment for patients with end-stage heart failure. While awaiting heart transplant, patients are typically medically managed with inotropic therapy which is aimed at increasing cardiac contractility and forward flow of arterial blood, thereby increasing oxygen delivery to the tissues. However, as left ventricular function continues to deteriorate, patients become more dependent upon intravenous inotropic therapy. ${ }^{[3]}$ Pre-heart transplant patients with congestive heart failure (New York Heart Association [NYHA] class IV) ${ }^{[4]}$ have few treatment modalities available. When pharmacologic therapies like diuretics, phosphodiesterase inhibitors

\footnotetext{
*Correspondence: Frederick R. Macapagal; Email: fmacapagal@houstonmethodist.org; Address: Houston Methodist Hospital 6565 Fannin St., Houston, Texas, United States.
} 
(i.e., milrinone), and adrenergic agonists (i.e., dobutamine, dopamine $)^{[5]}$ are ineffective, one mechanical supportive device option is an intra-aortic balloon pump (IABP).

The IABP is based on the counter pulsation principle. The gas-filled balloon (helium) at the tip of the IABP catheter located in the proximal descending thoracic aorta inflates during diastole, thereby displacing blood and increasing perfusion to the coronary arteries. The balloon deflates during systole, decreasing the afterload and increasing perfusion to the renal arteries supplying the kidneys, mesenteric arteries supplying the digestive organs, and distal arteries. ${ }^{[6,10]}$ The IABP provides excellent hemodynamic support while awaiting transplant. ${ }^{[7]}$

Arterial sites used for IABP insertion include the femoral artery, subclavian artery, axillary artery, ${ }^{[8]}$ ascending aorta through suprainguinal bypass grafts, and common iliac artery. ${ }^{[9]}$ The axillary, ascending aorta, and common iliac artery approaches require insertion in the operating room. In contrast, a femoral artery IABP can be inserted percutaneously at the bedside, rendering this approach the most commonly utilized. ${ }^{[10]}$ However, the femoral artery approach requires patients to remain in bed until a donor heart becomes available, which can take from 40 days or longer. ${ }^{[11]}$ The femoral artery approach is subsequently associated with significant risks and complications related to the effects of prolonged immobility.

Patients with a femoral artery IABP must remain relatively immobile in bed with the head of the bed at $\leq 30^{\circ}$ and no active flexion at the hip of the affected side. ${ }^{[12]}$ The detrimental effects of prolonged immobility result in severe widespread deconditioning, affecting multiple organs and systems. In the critical care setting, patients lose up to $30 \%$ muscle mass within 10 days of admission. ${ }^{[13]}$

Prolonged bedrest cause an overall decline in muscle mass, strength and function. ${ }^{[1]}$ Early physical therapy and mobility in the ICU setting should be safely implemented due to all the above listed reasons. ${ }^{[14]}$ Given that our pre heart transplant IABP patient population median wait time for a heart transplant is 25 days, the deleterious musculoskeletal effects of prolonged immobility can be profound if they had a femoral IABP.

A novel percutaneous left axillary-subclavian artery IABP insertion approach has been developed by cardiologists at our hospital to allow patients to mobilize out of bed while awaiting heart transplantation. The percutaneously placed axillary-subclavian intraaortic balloon pump (PAxIABP) is inserted in the cardiac catheterization laboratory under local anesthesia. This procedure is done with a direct axillary artery percutaneous stick using a $\mathbf{J}$ wire guide inserted into the left brachial artery or femoral artery into the axillarysubclavian artery as a roadmap. ${ }^{[15]}$ A regular intraaortic balloon pump catheter and console is used. The sheath and IABP catheter are sutured in place, secured with stat lock at the hub, and dressed with Tegaderm CHG (3M). The patient is then transferred to the Coronary Intensive Care Unit (CICU) where they remain until a donor heart becomes available. The PAIABP allows for increased physical activity, preventing the deleterious effects of prolonged immobility seen with a femoral-artery-inserted IABP. Patients are mobilized as soon as they are medically stable.

The specific aim of this retrospective study was to describe the level of mobilization and progress of PAIABP patients before heart transplantation. The preliminary hypothesis was that the patients could be mobilized after PAIABP placement while the patient's failing heart was supported.

\section{Patients and methods}

Hospital Institutional Review Board (IRB) approval was obtained prior to the start of this retrospective descriptive study. The study sample included 45 adult PAxIABP therapy patients awaiting heart transplantation in the CICU from January 2007 to May 2013. Inclusion criteria were as follows: patients aged 18 to 85 years, diagnosis of end-stage heart failure (NYHA class IIIb-IV), PAxIABP in place, and on the heart transplant list. Patients with lower extremity paralysis and those on mechanical ventilation were excluded from the study owing to their reduced ability to mobilize out of bed.

Data collection was by chart review. Data collection sheets were utilized by the co-investigators during chart review, after the investigators completed IRB training requirements. All data collected were then coded and secured in a locked cabinet in an office accessible only to the researchers to protect patient confidentiality.

The data collected included patient demographics (age, sex, body mass index, and race); comorbidities; daily review of mobilization and ambulation activity (a Pittsburgh Measuring Wheel and a nurse-developed chart assigning pre-measured distances between known 10th floor landmarks were used to measure distance walked by the patient); and physical therapist (PT) documentation. We also reviewed documented complications, inotropic drips, oxygen requirements, vital signs, hemodynamic pressure measurements, and total PAIABP support duration.

\subsection{Vital signs monitoring}

Vital signs monitoring during ambulation was initially obtained with portable transport monitors (GE Transport Pro) 
and the IABP console, but as the patient became more stable, monitoring was done by use of the IABP console (Datascope CS 300; Maquet) only. Patients' relatives were enlisted to help with mobilization and ambulation by pushing a standby wheelchair or IV poles for weaker patients .

Insertion site dressings were maintained per hospital policy with the use of chlorhexidine gluconate ( $\mathrm{CHG}$ )-impregnated Tegaderm (3M). Sutures, statlock anchors, and additional Tegaderm clear dressings were used to further secure the PAIABP.

Stable patients were allowed to sleep with minimal interruption at night. Laboratory tests and chest X-rays were done at 8 AM instead of the usual 4 to 5 AM. Therapeutic heparin infusions were monitored with anti-factor $\mathrm{Xa}$ assay levels per hospital protocol (every 6 hours until 2 therapeutic levels were achieved, then daily). Laboratory tests, like the basic metabolic panel and complete blood cell count, were done every other day to minimize risk of infection, blood loss, and the need for transfusion. ${ }^{[16]}$

Chest X-rays were done every day. If there was a need for repositioning of the PAIABP, it was done by the cardiologists at the bedside. Repeat chest X-rays were then done to confirm placement. Patients were taken to the cath lab if there was a need to replace the catheter or for more complicated repositioning.

\subsection{Functional independence measure}

The FIM is a physical therapy tool used to measure disability in acute care settings by applying scores based on level of assistance with activity. ${ }^{[17]}$ It provides a uniform system of measurement based on the International Classification of Impairment, Disabilities, and Handicaps. ${ }^{[18]}$ It is used to measure a patient's progress and assess rehabilitation outcomes. The complete scale includes 18 items that measure physical and cognitive disability and is useful in clinical settings of rehabilitation to assess outcomes, indicate the burden of caring for a patient, and assist in determining appropriate discharge destinations. Each item is scored from 1 to 7 based on the level of independence (where $1=$ total dependence and $7=$ complete independence).

FIM scores were obtained for the 1st, 2nd, 4th, and 6th PT sessions after PAxIABP placement. FIM scoring by PT was discontinued when the patient reached a score of 5 to 7 . Nurses took over managing patient mobilization when less PT assistance was needed and the patients gained the ability to mobilize more freely. However, PT still checked in with the nurses and patients occationally to offer assistance when new needs arose. Mobilization, for the purpose of our study, included dangling on the side of the bed, sitting up on a chair, and ambulating to the sink, bathroom, and throughout the CICU hallways and adjacent buildings. Special trips to the chapel, gardens, and the hospital lobby were allowed for stable patients with doctors' orders.

\subsection{Statistical analysis}

Data are presented as means (standard deviation) for continuous variables and as numbers (percentage) for categorical variables. All analyses were performed with STATA version 13 (StataCorp, College Station, TX).

\section{RESULTS}

Stratification was not done owing to the small number of test subjects. The patients' demographic characteristics and comorbidities are shown in Table 1. Thirty-seven patients $(82.2 \%)$ continued on PAxIABP therapy until heart transplantation. Two patients required LVAD implantation due to worsening heart failure and eventually received heart transplants. Six patients died while awaiting heart transplants, unrelated to the PAxIABP therapy. Six patients (13\%) had confirmed systemic or cardiac amyloidosis and had been denied heart transplantation in other institutions.

\subsection{Inotrope and oxygen requirements}

The most common inotropic medication used concurrently with PAxIABP therapy was milrinone (35\%), for which the dose was adjusted according to the patient's condition (range, $0.125-0.4 \mathrm{mcg} / \mathrm{kg} / \mathrm{min})$. Three patients $(6 \%)$ were receiving dopamine and one patient $(2 \%)$ was on dobutamine intermittently before transplant. Twenty-five patients (57\%) were not receiving any inotropes. Oxygen by nasal cannula (2-4 $1 / \mathrm{min}$ ) was used intermittently by 18 patients (40\%), but $60 \%$ $(n=27)$ were on room air or were weaned down to room air.

\subsection{Mobilization and ambulation}

Mobilization and ambulation included dangling on the side of the bed, getting up to the chair, walking to the sink and the bathroom. Ambulation included walking around the CICU unit, hospital hallways and on rare occasions, down to the indoor garden and fountain in the hospital lobby. The physical therapist (PT) intially evaluates the patient for mobility. The number of patients seen by PT decreased as patient mobility increased and nurses took over complete mobilization thereafter. Patients were mobilized on average $1.79( \pm 2.10)$ times daily. The time to initial mobilization after PAIABP insertion was $1.39( \pm 1.41)$ days, with 8 patients mobilized on the day of procedure. Average ambulation was every other day, with a maximum of 3 times per day for some patients. Mean distance travelled on ambulation was $1184.09( \pm 1558.57)$ feet. 
Table 1. Characteristics of the study population

\begin{tabular}{|c|c|c|c|}
\hline & Variable & $\mathbf{N}$ & $\%$ \\
\hline Age & & $56.1 \pm 10.2$ & \\
\hline \multirow[t]{2}{*}{ Gender } & Male & 35 & 77.80 \\
\hline & Female & 10 & 22.20 \\
\hline BMI & & $27.9 \pm 4.9$ & \\
\hline \multirow{5}{*}{ Race } & Caucasian & 28 & 62.2 \\
\hline & Black & 10 & 22.2 \\
\hline & Hispanic & 5 & 11.1 \\
\hline & Asian & 1 & 2.2 \\
\hline & Other & 1 & 2.2 \\
\hline \multirow{2}{*}{ Mortality } & Alive & 37 & 82 \\
\hline & Deceased & 8 & 18 \\
\hline BTT & & 45 & 100 \\
\hline \multirow{8}{*}{ Comorbidities } & Amyloidosis & 6 & 13 \\
\hline & CKD & 16 & 35 \\
\hline & Cardiomyopathy & 27 & 60 \\
\hline & D. Mellitus & 20 & 44 \\
\hline & COPD, Pulm. HTN & 17 & 38 \\
\hline & Heart Failure (NYHA Class 3b-4) & 45 & 100 \\
\hline & Previous Heart & 3 & 7 \\
\hline & Transplant & & \\
\hline \multirow{3}{*}{ Inotropes } & Milrinone & 16 & 35 \\
\hline & Dopamine & 3 & 6 \\
\hline & Dobutamine & 1 & 2 \\
\hline \multirow{2}{*}{ Oxygen } & Room air & 27 & 60 \\
\hline & Nasal cannula 1-4 l/min & 18 & 40 \\
\hline \multirow{5}{*}{ Vital signs } & Mean augmented diastolic & & $81.0 \pm 12.8$ \\
\hline & Systolic & & $95.5 \pm 14.5$ \\
\hline & Diastolic & & $57.5 \pm 12.5$ \\
\hline & MAP & & $76.2 \pm 11.24$ \\
\hline & Heart Rate & & $80.1 \pm 15.9$ \\
\hline \multirow{6}{*}{$\begin{array}{l}\text { Pulmonary artery } \\
\text { pressures }\end{array}$} & Systolic & & $57.9 \pm 11.91$ \\
\hline & Diastolic & & $26.97 \pm 2.49$ \\
\hline & Mean & & $38.55 \pm 2.53$ \\
\hline & Cardiac output & & $3.2 \pm .75$ \\
\hline & Cardiac index & & $1.58 \pm .32$ \\
\hline & Wedge & & $13.53 \pm 2.33$ \\
\hline
\end{tabular}

Note. BMI: Body Mass Index; BTT: bridge to transplant; CKD: chronic kidney disease; COPD: chronic obstructive pulmonary disease; Pulm. HTN: pulmonary hypertension; NYHA: New York Heart Association classification for heart failure; MAP: mean arterial pressure: A total of 6 patients had a pulmonary artery catheter (swan ganz catheter) inserted.

\subsection{PAIABP days}

The mean number of total PAxIABP days was $27( \pm 29)$. Total PAIABP days included post-transplant days when the PAxIABP was left in place for support. The PAxIABP was usually discontinued 2 days after heart transplant. Pre-transplant
PAxIABP days varied widely depending on the availability of donor organs ${ }^{[7]}$ (4 [9\%] patients were transplanted with $>1$ organs, i.e., heart-lung, heart-kidney), severity of comorbidities (6 [13\%] patients had cardiac and/or systemic amyloidosis), blood type compatibility, and patient size. 


\subsection{Complications}

Documented complications associated with this procedure and subsequent mobilization included limb ischemia complications (left arm numbness/pain, loss of left radial pulse; $7 \%, \mathrm{n}=4$ ), which were treated by repositioning or replacing the PAxIABP and giving pain medications. Line infection was noted in $2 \%(\mathrm{n}=1)$ of the patients and was treated with antibiotics. Bleeding at the insertion site was observed in $4 \%(\mathrm{n}=2)$ of the patients and was treated by stopping and or decreasing the heparin infusion dose (see Table 2). No data were gathered on malposition/exchange of the PAxIABP catheter owing to incomplete charting, although increased incidence of IABP catheter malposition was reported by Estep, 2013. ${ }^{[15]}$ Notable problems encountered during ambulation were 1 episode of IABP console shutdown (the console was plugged in at the nearest wall outlet) and 1 episode of ventricular tachycardia without loss of conciousness that resolved spontaneously. These complications were resolved without harm to the patient.

Table 2. Percutaneously inserted axillary subclavian IABP Mobilization Days, comparison of complications between PAxIABP vs. long term Femoral IABP support

\begin{tabular}{lll}
\hline & Mean & Range \\
\hline Pre-transplant PAIABP days & 24.98 & $2-147$ ( \pm 25.03 days) \\
Total PAIABP days & 27 & $4-182$ ( \pm 29 days) \\
Days to mobilization & 1.3 & $0 *-7$ \\
Times mobilized/day & 2 & $1-4$ \\
Times ambulated/day & 1 every other day & $1-3 /$ day \\
Distance ambulated & 1,205 feet & $2-9,980$ feet \\
Comparison of Complications & & \\
& PA $\times$ IABP & Long Term Femoral IABP \\
Bleeding & $4 \%$ & $4.6 \%$ (Cochran et al. 2002) \\
Ischemic complications & $7 \%$ & $8 \%-18 \%$ (Parissis, 2011) \\
Immobility/bedrest & 0 & $100 \%$ (bedrest restrictions) \\
Infection & $2 \%$ & $30 \%$ (Gjesdal et al. 2009) \\
\hline
\end{tabular}

*Day 0 is the day of insertion

\section{Discussion}

Pre-heart-transplant PAxIABP patients in this study were $100 \%$ mobilized as soon as their medical condition permitted, in contrast with the total bedrest associated with femoral IABP. This result was similar to a study by Tanaka et al. (95.5\% mobility), ${ }^{[8]}$ although their insertion technique differed and their goal was not exclusive to bridge to transplant. The PAxIABP complication rates were comparable to femoral IABP complications published in a literature review by Parissis. ${ }^{[19]}$ New nursing care protocols, staff education on IABP console management, and intensive interdisciplinary collaboration between PT, nursing, and the patients' families were instituted in caring for these patients. More research is needed on the preoperative psychological effect on patients (anecdotal accounts from multiple patients have been very positive) and postoperative implications of this alternative therapy. Other applications for this include procedures requiring long IABP therapy support like high risk percutaneous coronary intervention/ coronary artery bypass after a massive myocardial infarction, as a bridge to deci- sion making, and bridge to LVAD. Study limitations were the small sample size, results from a single hospital, and incomplete documentation on some patients.

\section{Significance OF THE STUdY}

This study shows that pre heart transplant patient with a percutaneously placed axillary subclavian intraaortic balloon pump can be safely mobilized thereby avoiding the complications of prolonged bedrest, as compared to complete bedrest for patients who have a traditional femoral intraaortic balloon pump.

\section{ACKNOWLEDGEMENTS}

Dr. Shannan Hamlin, PhD, RN, ACNP-BC, AGACNP-BC, CCRN; All the CICU staff for all their hard work with our patients; Dr. David Putney, PharmD; Dr. Jaya Paranilam, PhD, Dr. Jerry Estep, MD; Dr. Kevin Donahue, PharmD., Dr. Nena Bonuel, PhD., Jennifer Holmes,AMWA.

\section{CONFlicts of InTEREST Disclosure}

The authors declare that there is no conflict of interest. 


\section{REFERENCES}

[1] McKelvie RS, Moe G, Cheung A, et al. The 2011 Canadian Cardiovascular Society heart failure management guidelines update: focus on sleep apnea, renal dysfunction, mechanical circulatory support, and palliative care. Can J Cardiol. 2011; 27: 319-320. PMid:21601772 https://doi.org/10.1016/j.cjca.2011.03 .011

[2] Benjamin E, Blaha J, et al. Heart disease and stroke statistics-2017 update; a report from the American Heart Association. Circulation. 2017. https://doi.org/10.1161/CIR. 0000000000000485

[3] Hashim T, Sanam K, et al. Clinical Characteristics and Outcomes of Intravenous Inotropic Therapy in Advanced Heart Failure. 2015. ht tps://doi.org/10.1161/CIRCHEARTFAILURE. 114.001778

[4] The Criteria Committee of the New York Heart Association. Nomenclature and Criteria for Diagnosis of Diseases of the Heart and Great Vessels. 9th ed. Boston: Little, Brown \& Co; 1994; 253-256.

[5] Yancy CW, Jessup M, Bozkurt B, et al. 2013 ACCF/AHA guideline for the management of heart failure: executive summary [published online June 5, 2013]. Circulation. 2013; 128(16): 1810-1852. https://doi.org/10.1161/CIR.0b013e31829e8807

[6] Krishna M, Zacharowski K. Principles of intra-aortic balloon pump counterpulsation. Contin Educ Anaesth Crit Care Pain. 2009; 9(1): 24-28. https://doi.org/10.1093/bjaceaccp/mkn051

[7] Chen M. Mechanical circulatory assist devices. In: Woods L, Froelicher ES, Underhill Mozer S, Bridges EJ, eds. Cardiac Nursing. 6th ed. Baltimore, MD: Lippincott Williams \& Wilkins; 2010.

[8] Tanaka A, Tuladhar S, et al. The subclavian intraaortic balloon pump: A compelling bridge device for advanced heart failure. https://doi.org/10.1016/j.athoracsur.2015.05.087

[9] Garrett K, Grady KL. Intra aortic balloon pumping through the common iliac artery: management of the ambulatory intraaortic balloon pump patient. Prog Cardiovasc Nurs. 2000; 15(1): 14-20. https://doi.org/10.1111/j.0889-7204.2000.80383.x

[10] Ma P, Zhang Z, Song T, et al. Combining ECMO with IABP for the treatment of critically ill adult heart failure patients. Heart, Lung and Circulation. 2014; 23: 364. https://doi.org/10.1016/j.hlc. 2013.10.081
[11] Quader M, Wolfe L, Katlaps G, et al. Donor heart utilization following cardiopulmonary arrest and resuscitation: Influence of donor characteristics and wait times in transplant regions. Journal of Transplantation. 2014. https ://doi.org/10.1155/2014/519401

[12] Raman J, Loor G, London M, et al. Subclavian artery access for ambulatory balloon pump insertion. Ann Thorac Surg. 2010; 90(3): 1032 1034. https://doi.org/10.1016/j.athoracsur.2009.11.0 82

[13] Parry S, Puthucheary Z. The impact of extended bed rest on the musculoskeletal system in the critical care environment. Extrem Physiol Med. 2015; 4: 16. https://doi.org/10.1186/s13728-015-0 036-7

[14] Adler J, Daniel M. Early mobilization in the intensive care unit: a systematic review. Cardiopulmonary Physical Therapy Journal. 2012

[15] Estep J, Cordero-Reyes AM, Bhimaraj A, et al. Percutaneous placement of an intra-aortic balloon pump in the left axillary/subclavian position provides safe, ambulatory long-term support as bridge to heart transplantation. JACC Heart Fail. 2013; 1(5): 382-388. PMid:24621970 https ://doi.org/10.1016/j.jchf .2013.06 .002

[16] Scornik J, Bromberg J, Norman D, et al. An update on the impact of pre-transplant transfusions and allosensitization on time to renal transplant and on allograft survival. BMC Nephrology [serial online] Available from: MEDLINE Complete, Ipswich, MA. 2013.

[17] Keith RA, Granger CV, Hamilton BB, et al. The functional independence measure: a new tool for rehabilitation. Adv Clin Rehab. 1987; 1: 6-18. PMid:3503663

[18] World Health Organization. International Classification of Impairments, Disabilities, and Handicaps. A Manual of Classification Relating to the Consequences of Disease. Geneva: World Health Organization; 1980. Available from: http://apps.who.int/iris/ha ndle/10665/41003

[19] Parissis H, Soo A, Al-Alao B. Intraaortic balloon pump: literature review of risk factors related to complications of the intraaortic balloon pump. J Cardiothorac Surg. 2011; 6: 147. 